\title{
A STUDY ON THE ESTABLISHMENT OF FAST NEUTRON MULTIPLE MEASUREMENT EQUATION BASED ON LIQUID SCINTILLATION DETECTOR
}

\author{
YAO JUNPING LI XIAOJUN \& FAN SHOUXIANG
}

Research Inst. of Hi-Tech, Information Security Department, Xi'an, China

\begin{abstract}
The traditional theory and technology of neutron multi-weight measurement are becoming mature. The advantages of fast neutron measurement system make it possible to carry out more in-depth theoretical analysis of nuclear materials. In this paper, the derivation of the fast neutron multiplicity measurement equation based on liquid-flash is studied in detail; in particular the 4-order and 5-order fast-neutron multiplicity measurement equation is derived, which is correct and effective for the verification of the theory of establishing and solving the three following measurement equations. It is important to extend and expand the application range of the neutron multiplicity measurement technique, and to correct the additional measurement amount to eliminate the influence of the dead interval correction. If the high order multifunction of neutron provides important value for nuclear material control and accounting, then the establishment of neutron multiplex higher order equation is a step that must be taken, which plays the role of laying the foundation.

KEYWORDS: Traditional Theory and Technology of Neutron Multi-Weight Measurement
\end{abstract}

Received: Feb 06, 2019; Accepted: Feb 26, 2019; Published: May 25, 2019; Paper Id.: IJPRJUN20195

\section{INTRODUCTION}

Fast neutron measurement greatly reduces the negative effect of accidental coincidence in the related neutron test, which can appear in the sample at a high rate. Compared with the thermal neutron analysis system, fast neutron measurement allows for in-depth analysis of value-added phenomena in sample items. Finally, by reducing the measuring time and improving the measuring accuracy, the fast neutron experimental system has the advantages of reducing the burden on the equipment operators.

At present, several design goals during the development of the fast-neutron multiplicity counter based on the liquid-flash detector are in place. First, multiplex computing systems must have high detection efficiency to detect second-order, third-order, or even higher-order conformance. Second, electron dead time loss must be minimized to take advantage of the rapid attenuation of liquid flashes. Third, the system must also be able to measure the properties of complex plutonium with large ranges of variation. Finally, the geometry of the system should be insensitive to how the sample is placed in the counter.

The detection mechanism of fast neutron multiplicity measurement is different from that of slow neutron detection. Fast neutron multiplexing measurement can not completely meet the six basic assumptions of the traditional neutron multiplicity measurement and analysis model. However, when the fast neutron multiplicity measurement is used to estimate the sample parameters, the traditional neutron multiplicity measurement equation is still used, which may lead to relatively large errors. In fast neutron multiplicity measurement, because of the existence of scattering crosstalk, for a neutron, sometimes the detector will output two or even three neutron signals. Therefore, it is necessary to study the influence of scattering crosstalk on the measured data and put 
forward appropriate assumptions. Based on the analysis of the neutron transport mechanism, it is assumed that one neutron can cause more than two detector responses, and that each neutron has a probability of $\kappa$ after producing a signal in the first probe. Then the signal is generated in the second probe ( $\mathrm{k}$ can be called scattering crosstalk rate), but it does not cause the third detector response.

It is of great significance to study the calculation formula of fast neutron multiplex measurement based on liquid scintillation for studying the properties of nuclear components and improving the nuclear safety control technology.

\section{THE MATHEMATICAL BASIS}

(1) Probability generating function

Let $\mathrm{X}$ be a non-negative integer random variable, its distribution is listed as

$$
\begin{aligned}
& p_{k} \equiv P(X=k) \quad(k=0,1,2, \cdots) \\
& P(s)=\sum_{k=0}^{\infty} p_{k} s^{k} \text { is the probability generating. }
\end{aligned}
$$

(2) the k-order multiplier of the random variable $\mathrm{x}$ is equal to the k-order derivative at the place $\mathrm{P}(\mathrm{s})$ is in $\mathrm{S}=1$, that is

$$
E[X(X-1) \cdots(X-k+1)]=P^{(k)}(1), P^{(0)}(1)=P(1)=\sum_{k=0}^{\infty} p_{k}=1
$$

(3) The probability generating function $G_{z}(s)$ of the sum $Z=X_{1}+X_{2}+\cdots X_{n}$ of $\mathrm{n}$ independent nonnegative integer random variables $X_{1}, X_{2}, \cdots, X_{n}$ is equal to the product of , that is

$$
G_{z}(s)=G_{1}(s) G_{2}(s) \cdots G_{n}(s)
$$

When $X_{1}, X_{2}, \cdots, X_{n}$ have the same distribution, they also have the same probabilistic generating function $G(s)$, then the probability generating function of $Z=X_{1}+X_{2}+\cdots X_{n}$ is

$$
G_{z}(s)=[G(s)]^{n}
$$

(4) Let $X_{1}, X_{2}, \cdots, X_{n}$ be a series of independent and identically distributed nonnegative integer random variables, $\mathrm{N}$ is a non-negative integer value random variable independent of the sequence ${ }^{\left\{X_{n}\right\}}$, then the probability generating function of random variable $Y=X_{1}+X_{2}+\cdots+X_{N}$ is

$$
\begin{aligned}
& G_{Y}(s)=G_{N}[G(s)] \\
& G_{N}(z) \text { is the probability generating function of } N, G(s) \text { is the jointly distributed probability generating }
\end{aligned}
$$
function of $X_{i}(i=1,2, \cdots, n)$ 
According to the derivation formula of compound function, the factorial moment of one, two, three and four factorial of random variable $\mathrm{Y}$ is obtained.

$$
\begin{aligned}
& G_{Y}{ }^{\prime}(1)=\left.\frac{\mathrm{d} G_{N}[G(s)]}{\mathrm{d} G(s)} \frac{\mathrm{d} G(s)}{\mathrm{d} s}\right|_{s=1}=G_{N}{ }^{\prime}(1) G^{\prime}(1) \\
& G_{Y}^{\prime \prime}(1)=G_{N}^{\prime \prime}(1)\left[G^{\prime}(1)\right]^{2}+G_{N}{ }^{\prime}(1) G^{\prime \prime}(1) \\
& G_{Y}^{\prime \prime \prime}(1)=G_{N}^{\prime \prime \prime}(1)\left[G^{\prime}(1)\right]^{3}+3 G_{N}^{\prime \prime}(1) G^{\prime \prime}(1) G^{\prime}(1)+G_{N}^{\prime}(1) G^{\prime \prime \prime}(1) \\
& G_{Y}{ }^{\prime \prime \prime}(1)= \\
& G_{N}{ }^{\prime \prime \prime}(1)\left[G^{\prime}(1)\right]^{4}+6 G_{N}{ }^{\prime \prime \prime}(1) G^{\prime \prime}(1)\left[G^{\prime}(1)\right]^{2}+4 G_{N}{ }^{\prime \prime}(1) G^{\prime \prime \prime}(1) G^{\prime}(1)(5) \text { The first, second and third moments of binomial } \\
& +3 G_{N} "(1)\left[G^{\prime \prime}(1)\right]^{2}+G_{N}{ }^{\prime}(1) G^{\prime \prime \prime}(1)
\end{aligned}
$$

distribution are respectively

$$
\begin{aligned}
& \sum_{i=1}^{n} i\left(\begin{array}{l}
n \\
i
\end{array}\right) p^{i}(1-p)^{n-i}=n p \\
& \sum_{i=2}^{n} i(i-1)\left(\begin{array}{c}
n \\
i
\end{array}\right) p^{i}(1-p)^{n-i}=n(n-1) p^{2} \\
& \sum_{i=3}^{n} i(i-1)(i-2)\left(\begin{array}{c}
n \\
i
\end{array}\right) p^{i}(1-p)^{n-i}=n(n-1)(n-2) p^{3}
\end{aligned}
$$

(6) Exponential integral

The original function of $f(t)=\frac{1}{\tau} e^{-\frac{t}{\tau} \text { is }} f(t)=-e^{-\frac{t}{\tau}}+C$

$$
\begin{gathered}
f_{d}=2 \int_{0}^{\infty}\left[f(t) \int_{t+P D}^{t+P D+G} f(s) \mathrm{d} s\right] \mathrm{d} t=e^{-P D / \tau}\left(1-e^{-G / \tau}\right) \quad f_{t}=3 \int_{0}^{\infty} f(t)\left[\int_{t+P D}^{t+P D+G} f(s) d s\right]^{2} d t=\left[e^{-P D / \tau}\left(1-e^{-G / \tau}\right)\right]^{2} \\
f_{q}=4 \int_{0}^{\infty} f(t)\left[\int_{t+P D}^{t+P D+G} f(s) d s\right]^{3} d t=\left[e^{-P D / \tau}\left(1-e^{-G / \tau}\right)\right]^{3} f_{\mathrm{p}}=5 \int_{0}^{\infty} f(t)\left[\int_{t+P D}^{t+P D+G} f(s) d s\right]^{4} d t=\left[e^{-P D / \tau}\left(1-e^{-G / \tau}\right)\right]^{4}
\end{gathered}
$$

\section{DERIVATION OF THE FAST NEUTRON MULTIPLICITY MEASUREMENT EQUATION}

It can be seen from the derivation of the traditional neutron multiplicity equation[3] that the distribution of the neutron number of the samples is independent of the detector. Directly use

$$
\begin{aligned}
& H^{\prime}(1)=\frac{F v_{s f, 1}}{F+F_{\alpha}}(1+\alpha) M \\
& H^{\prime \prime}(1)=M^{2} \frac{F}{F+F_{\alpha}}\left(v_{s f, 2}+v_{s f, 1}(1+\alpha) v_{i 2} \frac{M-1}{v_{i 1}-1}\right) \\
& H^{\prime \prime \prime}(1)=M^{3} \frac{F}{F+F_{\alpha}}\left(v_{s f, 3}+3 v_{s f, 2} v_{i 2} \frac{M-1}{v_{i 1}-1}+v_{s f, 1}(1+\alpha) v_{i 3} \frac{M-1}{v_{i 1}-1}+3 v_{s f, 1}(1+\alpha) v_{i 2}{ }^{2}\left(\frac{M-1}{v_{i 1}-1}\right)^{2}\right) \text { After the derivation, }
\end{aligned}
$$




$$
\begin{aligned}
& H^{\prime \prime \prime}(1)= M^{4} \frac{F}{F+F_{\alpha}}\left(v_{s f, 4}+6 v_{s f, 3} v_{i 2} \frac{M-1}{v_{i 1}-1}+4 v_{s f, 2} v_{i 3} \frac{M-1}{v_{i 1}-1}+15 v_{s f, 2} v_{i 2}^{2}\left(\frac{M-1}{v_{i 1}-1}\right)^{2}+\right.\text { is obtained. } \\
&\left.v_{s f, 1}(1+\alpha) \frac{M-1}{v_{i 1}-1}\left(v_{i 4}+10 v_{i 2} v_{i 3} \frac{M-1}{v_{i 1}-1}+15 v_{i 2}^{3}{ }_{i 2}\left(\frac{M-1}{v_{i 1}-1}\right)^{2}\right)\right)
\end{aligned}
$$

(2) Considering the effect of scattering crosstalk, the probabilistic generating function of the number of signals produced by a sample emitted neutrons should be

$e(z)=(1-\varepsilon)+\varepsilon(1-\kappa) z+\varepsilon \kappa z^{2}$

(3) The probabilistic generating function of the number of signals generated by a source event is

$$
d(z)=H(e(z))
$$

The first four factorial moments of a primary event to detect neutron weights are

$$
\begin{gathered}
d^{\prime}(1)=H^{\prime}(1) e^{\prime}(1)=\varepsilon(1+\kappa) H^{\prime}(1) \\
d^{\prime \prime}(1)=H^{\prime \prime}(1)\left(e^{\prime}(1)\right)^{2}+H^{\prime}(1) e^{\prime \prime}(1)=\varepsilon^{2}(1+\kappa)^{2} H^{\prime \prime}(1)+2 \varepsilon \kappa H^{\prime}(1) \\
d^{\prime \prime \prime}(1)=H^{\prime \prime \prime}(1)\left(e^{\prime}(1)\right)^{3}+3 H^{\prime \prime}(1) e^{\prime \prime}(1) e^{\prime}(1)+H^{\prime}(1) e^{\prime \prime \prime}(1) \\
=\varepsilon^{3}(1+\kappa)^{3} H^{\prime \prime \prime}(1)+6 \varepsilon^{2} \kappa(1+\kappa) H^{\prime \prime}(1) \\
d^{\prime \prime \prime}(1)=H^{\prime \prime \prime}(1) \varepsilon^{4}(1+\kappa)^{4}+12 H^{\prime \prime \prime}(1) \varepsilon^{3} \kappa(1+\kappa)^{2}+12 H^{\prime \prime}(1) \varepsilon^{2} \kappa^{2} \\
d^{\prime \prime \prime}(1)=H^{\prime \prime \prime}(1) \varepsilon^{4}(1+\kappa)^{4}+12 H^{\prime \prime \prime}(1) \varepsilon^{3} \kappa(1+\kappa)^{2}+12 H^{\prime \prime}(1) \varepsilon^{2} \kappa^{2}
\end{gathered}
$$

(4) Assuming that the source event occurs at the moment $t=0$, the time distribution of the detected neutron signal is $f(t)$, the counting gate width is $\mathrm{G}$, and the pre-delay is PD, then the true coincidence multiplicity of one, two, and three global moments is derived as follows:

$$
\begin{aligned}
r_{\text {1总 }}= & \frac{d^{\prime \prime}(1)}{d^{\prime}(1)} \int_{0}^{\infty}\left[f(t) \int_{t+P D}^{t+P D+G} f(s) \mathrm{d} s\right] \mathrm{d} t \\
= & \frac{\frac{1}{2} \varepsilon^{2}(1+\kappa)^{2} H^{\prime \prime}(1)+\varepsilon \kappa H^{\prime}(1)}{d^{\prime}(1)} f_{d} \\
r_{\text {2总 }}= & \frac{d^{\prime \prime \prime}(1)}{d^{\prime}(1)} \int_{0}^{\infty} f(t)\left[\int_{t+P D}^{t+P D+G} f(s) d s\right]^{2} d t \\
= & \frac{\frac{1}{3} \varepsilon^{3}(1+\kappa)^{3} H^{\prime \prime \prime}(1)+2 \varepsilon^{2} \kappa(1+\kappa) H^{\prime \prime}(1)}{d^{\prime}(1)} f_{t} \\
r_{3 \text { 总 }}= & \frac{d^{\prime \prime \prime}(1)}{d^{\prime}(1)} \int_{0}^{\infty} f(t)\left[\int_{t+P D}^{t+P D+G} f(s) d s\right]^{3} d t \\
& \frac{\frac{1}{4} \varepsilon^{4}(1+\kappa)^{4} H^{\prime \prime \prime}(1)+3 \varepsilon^{3} \kappa(1+\kappa)^{2} H^{\prime \prime \prime}(1)+3 \varepsilon^{2} \kappa^{2} H^{\prime \prime}(1)}{d^{\prime}(1)} f_{q}
\end{aligned}
$$

(5) The detected neutrons not only contain the neutrons produced by the source events directly or indirectly, but also contain the background neutrons which are independent of the source events. Mark $f_{i}(i=1,2,3)$ as the factorial 
moment of foreground multiplicity, $b_{i}(i=1,2,3)$ is coincidence multiplicity. The foreground counter is triggered by detected neutrons and the total trigger rate is

$$
S=\left(F+F_{\alpha}\right) d^{\prime}(1)+S_{b k g}
$$

The first, second, and third moments of the sample of true coincidence multiplicity are derived as follows:

$$
\begin{aligned}
& r_{1 \text { 样 }}=\frac{S\left(f_{1}-b_{1}\right)}{\left(F+F_{\alpha}\right) d^{\prime}(1)} \\
& r_{2 \text { 样 }}=\frac{S}{\left(F+F_{\alpha}\right) d^{\prime}(1)}\left[\left(f_{2}-b_{2}\right)-2 b_{1}\left(f_{1}-b_{1}\right)\right] \\
& r_{3 \text { 样 }}=\frac{S}{\left(F+F_{\alpha}\right) d^{\prime}(1)}\left[\left(f_{3}-b_{3}\right)-3 b_{1}\left(f_{2}-b_{2}\right)-3 b_{2}\left(f_{1}-b_{1}\right)+6 b_{1}^{2}\left(f_{1}-b_{1}\right)\right]
\end{aligned}
$$

Make the total moment equal to the

sample moment, and let

$$
\begin{aligned}
& \text { Singles }=S-S_{b k g} \\
& \text { Doubles }=S\left(f_{1}-b_{1}\right) \text { Triples }=\frac{1}{2} S\left[\left(f_{2}-b_{2}\right)-2 b_{1}\left(f_{1}-b_{1}\right)\right] \\
& \text { Quarters }=\frac{1}{6} S\left[\left(f_{3}-b_{3}\right)-3 b_{1}\left(f_{2}-b_{2}\right)-3 b_{2}\left(f_{1}-b_{1}\right)+6 b_{1}\left(f_{1}-b_{1}\right)^{2}\right] \text { then } \\
& \text { Singles }=F \varepsilon(1+\kappa) v_{s f, 1}(1+\alpha) M \\
& \text { Doubles }=\frac{F \varepsilon^{2}(1+\kappa)^{2} f_{d} M^{2}}{2}\left(v_{s f, 2}+v_{s f, 1}(1+\alpha) v_{i 2}\left(\frac{M-1}{v_{i 1}-1}\right)\right) \\
& +F \varepsilon \kappa f_{d} v_{s f, 1}(1+\alpha) M \\
& \text { Triples }=\frac{F \varepsilon^{3}(1+\kappa)^{3} f_{t} M^{3}}{6}\left(v_{s f, 3}+3 v_{s f, 2} v_{i 2}\left(\frac{M-1}{v_{i 1}-1}\right)+v_{s f, 1}(1+\alpha) v_{i 3}\left(\frac{M-1}{v_{i 1}-1}\right)\right. \\
& \left.+3 v_{s f, 1}(1+\alpha) v_{i 2}^{2}\left(\frac{M-1}{v_{i 1}-1}\right)^{2}\right) \\
& +F \varepsilon^{2} \kappa(1+\kappa) f_{t} M^{2}\left(v_{s f, 2}+v_{s f, 1}(1+\alpha) v_{i 2}\left(\frac{M-1}{v_{i 1}-1}\right)\right) \\
& \text { Quarters }=\frac{F \varepsilon^{4}(1+\kappa)^{4} f_{q} M^{4}}{24}\left(v_{s f, 4}+6 v_{s f, 3} v_{i 2}\left(\frac{M-1}{v_{i 1}-1}\right)+4 v_{s f, 2} v_{i 3}\left(\frac{M-1}{v_{i 1}-1}\right)+\right. \\
& \left.15 v_{s f, 2} v^{2}{ }_{i 2}\left(\frac{M-1}{v_{i 1}-1}\right)^{2}+v_{s f, 1}(1+\alpha)\left(\frac{M-1}{v_{i 1}-1}\right)\left(v_{i 4}+10 v_{i 2} v_{i 3}\left(\frac{M-1}{v_{i 1}-1}\right)+15 v^{3}{ }_{i 2}\left(\frac{M-1}{v_{i 1}-1}\right)^{2}\right)\right)+ \\
& \frac{F \varepsilon^{3}(1+\kappa)^{2} k f_{q} M^{3}}{2}\left(v_{s f, 3}+3 v_{s f, 2} v_{i 2}\left(\frac{M-1}{v_{i 1}-1}\right)+v_{s f, 1}(1+\alpha) v_{i 3}\left(\frac{M-1}{v_{i 1}-1}\right)+\right. \\
& \left.3 v_{s f, 1}(1+\alpha) v_{i 2}^{2}\left(\frac{M-1}{v_{i 1}-1}\right)^{2}\right)+\frac{F \varepsilon^{2} k^{2} f_{q} M^{2}}{2}\left(v_{s f, 2}+v_{s f, 1}(1+\alpha) v_{i 2}\left(\frac{M-1}{v_{i 1}-1}\right)\right)
\end{aligned}
$$

\section{DERIVATION PROCESS ANALYSIS}

Compared with the derivation process of traditional neutron multiplicity measurement formula and fast neutron multiplicity measurement formula, first of all, the probability generating function of the number of signals produced by 
neutron emission from a sample in fast neutron measurement should be as follows.

$$
e(z)=(1-\varepsilon)+\varepsilon(1-\kappa) z+\varepsilon \kappa z^{2}
$$

In conventional neutron measurement, the probability generating function of the number of signals emitted from a sample should be

$$
e(z)=(1-\varepsilon)+\varepsilon z
$$

Although the complexity degree of one is linear and the other is quadratic, they have a great influence on the multiplicity expression of neutron emission from the sample, especially when pushing down the quadruple is quite complicated.

According to the previous study, it is not difficult to sum up the derivation method of the fast neutron's quaternion (pents or 5th order).

Here's our formula for quaternion measurement

$$
\begin{gathered}
\text { Pents }=\frac{F \varepsilon^{5}(1+k)^{5} f_{p} M^{5}}{120}\left(V_{s f, 5}+\left(V_{s f, 1}(1+\alpha) V_{i 5}+\right.\right. \\
\left.5 V_{s f, 2} V_{i 4}+10 V_{s f, 3} V_{i 3}+10 V_{s f, 4} V_{i 2}\right)\left(\frac{M-1}{V_{i 1}-1}\right)+\left(V_{s f, 1}(1+\alpha)\right. \\
\left.\left.\left(15 V_{i 2} V_{i 4}+10 V_{i 2}{ }^{3}\right)+60 V_{s f, 2} V_{i 2} V_{i 3}+45 V_{s f, 3} V_{i 2}{ }^{2}\right)\left(\frac{M-1}{V_{i 1}-1}\right)^{2}+105 V_{s f, 1}(1+\alpha) V_{i 2}{ }^{2} V_{i 3}+105 V_{s f, 2} V_{i 2}{ }^{3}\right)\left(\frac{M-1}{V_{i 1}-1}\right)^{3}+ \\
105 V_{s f, 1}(1+\alpha) V_{i 2}{ }^{4}\left(\frac{M-1}{V_{i 1}-1}\right)^{4}+\frac{F \varepsilon^{4}(1+K)^{3} k f_{p} M^{4}}{6} \\
\left(V_{s f, 4}+6 V_{s f, 3} V_{i 2} \frac{M-1}{v_{I 1}-1}+4 V_{s f, 2} V_{i 3} \frac{M-1}{v_{I 1}-1}+15 V_{s f, 2} V_{i 2}{ }^{2}\right) \\
\left(\frac{M-1}{V_{i 1}-1}\right)^{2}+V_{s f, 1}(1+\alpha) \frac{M-1}{V_{i 1}-1}\left(V_{i 4}+10 V_{i 2} V_{i 3}+\right. \\
\left.\left.\frac{M-1}{V_{i 1}-1} 15 V_{i 2}{ }^{3}\left(\frac{M-1}{V_{i 1}-1}\right)^{2}\right)\right)+\frac{F \varepsilon^{3}(1+K) k^{2} f_{p} M^{3}}{2} \\
\left(V_{s f, 3}+3 V_{s f, 2} V_{i 2}\left(\frac{M-1}{V_{i 1}-1}\right)+V_{s f, 1}(1+\alpha) V_{i 3}\left(\frac{M-1}{V_{i 1}-1}\right)+3 V_{s f, 1}(1+\alpha) V_{i 2}{ }^{2}\left(\frac{M-1}{V_{i 1}-1}\right)^{2}\right)
\end{gathered}
$$

In fact, the above derivation process can be carried on continuously, and the formula of multiplicity measurement of any order can be obtained finally. [3]. 


\section{CONCLUSIONS}

In this paper, for the first time, the quaternion measurement equations and quaternion measurement equations of fast neutrons are given, which play an important role in the study of the measurements that are only used in triples before correction. If the future of high order multiplicity can provide important value for nuclear material control and accounting, the formula method presented here will play a role in laying the foundation.

\section{REFERENCES}

1. N. Ensslin, W.C. Harker, M.S. Krick, D.G. Langner, M.M. Pickrell, J.E. Stewart, Application Guide to Neutron Multiplicity Counting. Los Alamos Report LA-13422-M (1998)

2. W. Hage and D.M. Cifarelli, "On the Factorial Moments of the Neutron Multiplicity Distribution of Fission Cascades", Nucl. Instr. Meth. A 236, pp. 165 (1985).

3. I. P'azsit and L. P'al, Neutron Fluctuations - a Treatise on the Physics of Branching Processes. Elsevier Science Ltd, London, New York, Tokyo, 2008

4. Richard A. Brualdi 著, 冯速等译. 组合数学. 机械工业出版社, 2013 年4 月, P134-136.

5. 《数学手册》编写组. 数学手册. 高等教育出版社, 1979 年, P792-793.

6. 许小明. 中子多重性测量技术研究, 博士论文, TL249.3, 2008.07 .

7. NEUTRON FLUCTUATIONS, Chamers University of Technology, Gothenburg,Sweden. Elsevier, The Boulevard, Landford Lane,Kidlington,Oxford OX5 1GB, UK Radarweg 29, PO Box 211,1000 AE Amsterdam, The Netherlands,First edition 2008.

8. D.L.Chichester,etc. IMPACT Fast Neutron Multiplicity System Prototype Development. 2013.09, INL is a U.S. Department of Energy National Laboratory operated by Battelle Energy Allianc.

9. Nakae, L.;G. Chapline;A. Glenn et al., (2014). The use of fast neutron detection for materials accountability. International Journal of Modern Physics: Conference Series, World Scientific.

10. Chichester, D.;S. Pozzi;J. Dolan et al., (2012). "MPACT Fast Neutron Multiplicity System Design Concepts." Idaho National Laboratory, Idaho Falls, ID.

11. Chichester, D. L.;S. J. Thompson; M. T. Kinlaw et al. (2015)"Statistical estimation of the performance of a fast-neutron multiplicity system for nuclear material accountancy." Nuclear Instruments and Methods in Physics Research Section A: Accelerators, Spectrometers, Detectors and Associated Equipment 784(0): 448-454.

12. Andrea Favalli, Stephen Croft, Peter Santi. Point model equations for neutron correlation counting: Extension of Böhnel's equations to any order [J]. Nuclear Instruments and Methods in Physics Research A795 (2015) 370-375. 
